Second, how does Ubx2 recognize that a precursor protein is stuck in the TOM complex? Ubx 2 can bind to ubiquitin, so ubiquitin is probably used to tag clogged precursor proteins. Defining the components of the ubiquitination machinery and the ubiquitin signals that govern not just the mitoTAD pathway, but also the stress-induced quality-control pathways that regulate mitochondrial protein import will be an important direction for future research. The fact that the ER and mitochondria use the same protein for quality control might imply that these processes are co-regulated.

Many other fascinating questions also remain to be addressed. In the meantime, Mårtensson and colleagues' work has painted an illuminating picture of a previously unknown aspect of how mitochondria protect their main entry gate.

Sylvie Callegari and Peter Rehling are in

the Department of Cellular Biochemistry,

University Medical Center Göttingen, 37073

Göttingen, Germany. P.R. is also at the Max

Planck Institute for Biophysical Chemistry,

Göttingen.

e-mail:peter.rehling@medizin.uni-goettingen.de

1. Mårtensson, C. U. et al. Nature 569, 679-683 (2019).

2. Pfanner, N., Warscheid, B. \& Wiedemann, N. Nature Rev. Mol. Cell Biol. 20, 267-284 (2019).

3. Wrobel, L. et al. Nature 524, 485-488 (2015).

4. Weidberg, H. \& Amon, A. Science 360, eaan4146 (2018).

5. Boos, F. et al. Nature Cell Biol. 21, 442-451 (2019).

6. Wang, X. \& Chen, X. J. Nature 524, 481-484 (2015).

7. Gregersen, N., Bross, P., Vang, S. \& Christensen,
J. H. Annu. Rev. Genomics Hum. Genet. 7, 103-124 (2006).

8. Mehnert, C. S. et al. J. Biol. Chem. 289, 27352-27362 (2014).

9. Ast, T., Michaelis, S. \& Schuldiner, M. Cell 164 103-114 (2016).

10.Neuber, O., Jarosch, E., Volkwein, C., Walter, J. \& Sommer, T. Nature Cell Biol. 7, 993-998 (2005).

11.Schuberth, C. \& Buchberger, A. Nature Cell Biol. 7, 999-1006 (2005).

12.van der Laan, M. et al. Mol. Cell Biol. 25, 7449-7458 (2005).

13.Schendzielorz, A. B. et al. J Cell Bio/ 216, 83-92 (2017).

14.Izawa, T., Park, S.-H., Zhao, L., Hartl, F. U. \& Neupert, W. Cell 171, 890-903 (2017)

15. Heo, J.-M. et al. Mol. Cell 40, 465-480 (2010).

16.Okreglak, V. \& Walter, P. Proc. Natl Acad. Sci. USA 111, 8019-8024 (2014).

17.Chen, Y.-C. et al. EMBO J. 33, 1548-1564 (2014).

This article was published online on 22 May 2019.

\title{
Brain cells invade prostate tumours
}

Prostate cancer contains nerve cells that are linked to disease progression, but their source was unknown. A mouse study reveals that cells from the brain invade prostate tumours and give rise to this nerve-cell population. SEE ARTICLE P.672

\section{SIMON T. SCHAFER \& FRED H. GAGE}

$\mathrm{M}$ any studies ${ }^{1-6}$ in the past decade have shown that interactions between tumour cells and nerve cells can influence the progression of various types of cancer. In prostate tumours, the presence of newly formed nerve cells is associated with the progression of malignancy and tumour spread to other locations in the body ${ }^{1,2,7}$. The microenvironment of a tumour can permit the development of such new nerve cells in a process called neo-neurogenesis. Yet how these nerve cells emerge in tumours in the first place has been a key unanswered question. Writing in Nature, Mauffrey et al. ${ }^{8}$ report a study in mice which indicates that a type of precursor cell called a neural progenitor cell, from a region of the brain in which the normal process of nerve-cell formation (neurogenesis) occurs, migrates to the site of prostate cancer. These cells invade the tumour microenvironment and give rise to new nerve cells.

The processes by which embryos and solid tumours develop are thought to share many features. In most multicellular animals, the nervous system and the vasculature have roles in both of these processes. In mice, neurons arise throughout life, by neurogenesis from neural progenitor cells in two regions of the adult brain - the subventricular zone and the dentate gyrus ${ }^{9}$. Although there has been controversy lately about the extent of neurogenesis

in adult humans ${ }^{10-12}$, decades of previous work have indicated that humans generate new neurons throughout the course of life only in the dentate gyrus ${ }^{13,14}$.

To address the origin of nerve cells in prostate tumours, Mauffrey and colleagues used a mouse model of prostate cancer called Hi-myc. The authors report that prostate tumours in these animals were associated with a type of newborn nerve cell called a neuroblast (Fig. 1). These cells express the protein doublecortin

"Neural progenitor cells from the subventricular zonecan migrate from the brain." (DCX), which is also expressed by special types of neural progenitor cell, which give rise to neurons.

The authors isolated DCX-expressing cells (which might be neural progenitor cells or neuroblasts) from samples of the animals' prostate tumours, and analysed the expression of genes and proteins. Using an approach termed antigenic profiling, they tracked the source of the DCX-expressing neuroblast cells that they had isolated, and found that the cells expressed several proteins characteristic of neural progenitor cells that can give rise to neurons. The neuroblasts did not express the gene $M y c$, which drives prostate tumour formation in the Hi-myc model, leading the authors to conclude that these neuroblasts were probably not derived from tumour cells. Further neuroblast-cell profiling did not reveal any obvious similarities to other types of cell in the tumour microenvironment, such as immune or endothelial cells. The authors found that the tumour-associated neuroblasts had molecular hallmarks similar to those of neural progenitor cells from the subventricular zone. Moreover, the cells could differentiate into neuronal-like cells when grown in vitro.

Mauffrey and colleagues next studied neural progenitor cells in the mouse brain, obtaining crucial support for their proposal that these cells drive neo-neurogenesis in prostate tumours. The authors tracked the cells using a technique that labels neural progenitor cells in specific brain regions. This revealed that neural progenitor cells from the subventricular zone can migrate from the brain and travel through blood vessels to specifically invade prostate tumours. However, the molecular factors and the mechanisms that guide the migration of these cells to this location are unknown. The authors found no evidence that neural progenitor cells from the dentate gyrus exited the brain. And they report that there was a selective breach in the blood-brain barrier around the region of the subventricular zone, but that no such breach was associated with the dentate gyrus.

To determine the relevance of their findings to other types of cancer, Mauffrey and colleagues used various mouse cancer models generated through the transplantation of human tumour cells. The authors consistently observed a similar mode of neural-progenitorcell migration from the brain to the tumours. Moreover, when they used a technique to produce mice that had selective depletion of DCX-expressing neural progenitor cells and neuroblasts, the resulting animals showed impaired tumour establishment compared with control mice. When mice bearing human prostate tumours were transplanted with neuroblasts from either mouse prostate 


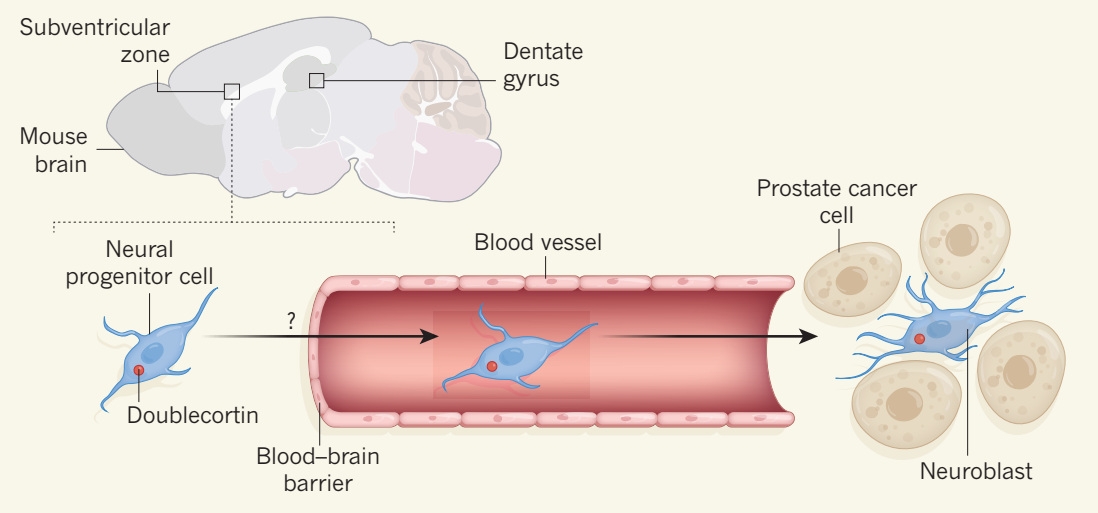

Figure 1 | Nerve cells in prostate tumours arise from brain cells. The formation of nerve cells in the adult mouse brain occurs in the subventricular zone and the dentate gyrus. Mauffrey et al. ${ }^{8}$ report that, in a mouse model of prostate cancer, there is a breach in the blood-brain barrier near the subventricular zone that is caused by unknown factors. Neural progenitor cells from the subventricular zone exit the brain through this breach, migrate through blood vessels and specifically invade prostate tumours. At the site of the cancer, the neural progenitor cells, which express the protein doublecortin (a marker protein that the authors used to track the development of neuronal cell populations), give rise to a type of immature nerve cell called a neuroblast, revealing the origins of newly formed nerve cells in the tumour microenvironment that are linked to tumour progression ${ }^{1,2,7}$.

tumours or mouse neural progenitor cells from the subventricular zone, the animals in both of these groups showed enhanced tumour growth and tumour spread to other parts of the body compared with mice that did not receive transplants. Mauffrey and colleagues also found that an increased density of neuroblasts seen in prostate samples from people with prostate cancer correlated with greater aggressiveness and swifter recurrence of these individuals' tumours.

The authors' findings suggest a previously unsuspected and intriguing role for brainderived neuroblasts in influencing tumour development and progression. However, more investigation will be needed to address the key question of whether this phenomenon has clinical relevance for human prostate cancer. Furthermore, it remains to be determined convincingly that neurogenesis in humans normally persists in the subventricular zone throughout life, so it might be that the mechanisms that drive neo-neurogenesis in humans are different from those observed in the systems studied by Mauffrey and colleagues. In addition, the Hi-myc mouse model might not be sufficient to model all types of prostate cancer. If that is the case, it would be useful to test other mouse models, to determine whether these findings reflect a general mechanism or one that is specific to this particular model.

Regardless of such unknowns, Mauffrey and colleagues' study undoubtedly provides one explanation for the origins of newly formed nerve cells in prostate tumours in mice. But the possibility that some of these nerve cells arise spontaneously from other types of cell in the tumour or its surroundings cannot be ruled out. Nevertheless, the authors' findings warrant further research to illuminate the details of the mechanisms responsible. For example, how is a breach in the blood-brain barrier initiated in a way that is specific to the subventricular zone? The answers to this and other questions should help to shed light on this fascinating topic. $\mathrm{a}$

Simon T. Schafer and Fred H. Gage are at The Salk Institute for Biological Studies, La Jolla, California 92037, USA.

e-mails:sschafer@salk.edu; gage@salk.edu

1. Ayala, G. E. et al. Clin. Cancer Res. 14, 7593-7603 (2008).

2. Magnon, C. et al. Science 341, 1236361 (2013).

3. Zhao, C. M. et al. Sci. Transl. Med. 6, 250ra115 (2014).

4. Stopczynski, R. E. et al. Cancer Res. 74, 1718-1727 (2014).

5. Peterson, S. C. et al. Cell Stem Cell 16, 400-412 (2015).

6. Hayakawa, Y. et al. Cancer Cell 31, 21-34 (2017).

7. Dobrenis, K., Gauthier, L. R., Barroca, V. \& Magnon, C. Int. J. Cancer 136, 982-988 (2015).

8. Mauffrey, P. et al. Nature https://doi.org/10.1038/ s41586-019-1219-y (2019).

9. Gonçalves, J. T., Schafer, S. T. \& Gage, F. H. Cell 167, 897-914 (2016).

10.Kempermann, G. et al. Cell Stem Cell 23, 25-30 (2018).

11.Moreno-Jiménez, E. P. et al. Nature Med. 25, 554-560 (2019).

12.Sorrells, S. F. et al. Nature 555, 377-381 (2018).

13. Eriksson, P. S. et al. Nature Med. 4, 1313-1317 (1998).

14.Spalding, K. L. et al. Cell 153, 1219-1227 (2013).

\title{
A step forward for artificial touch systems
}

\begin{abstract}
Object manipulation using an innovative glove allows large databases of detailed pressure maps to be obtained. Such data could lead to advances in robotic sensing and in our understanding of the role of touch in manipulation. SEE LETTER P.698
\end{abstract}

\section{GIULIA PASQUALE}

$\mathrm{T}$ The study and replication of human sensory abilities, such as visual, auditory and tactile (touch-based) perception, depend on the availability of suitable data. Generally, the larger and richer the data set, the more closely models can mimic these functions. Advances in artificial visual and speech systems rely on powerful models, known as deep-learning models, and have been fuelled by the ubiquity of databases of digital images and spoken audio (see, for example, go.nature.com/2w7nc0q). By contrast, progress in the development of tactile sensors - devices that convert a stimulus of physical contact into a measurable signal - has been limited, mainly because of the difficulty of integrating electronics into flexible materials ${ }^{1}$. On page 698, Sundaram et al. ${ }^{2}$ report their use of a low-cost tactile glove that addresses this issue.

The authors' glove consists of a hand-shaped sensing sleeve that is attached to the palm side of a knitted glove (Fig. 1). The sleeve contains a force-sensitive film on which is sewn a network of 64 electrically conducting threads: 32 along one direction of the glove and 32 along the perpendicular direction. Each of the 548 points at which these threads overlap is a pressure sensor, because the electrical resistance of the interleaved film decreases when these points are pressed. The output of the glove can be processed as a $32 \times 32$ array of greyscale pixels, in which the colour of each pixel indicates the applied pressure from low (black) to high (white). These pressure maps are recorded at about seven frames per second.

In Sundaram and colleagues' study, the glove was worn to record several videos of pressure maps during 3-5-minute sessions 